Arqueología y Sociedad,

№ 20, 2009

\title{
ANÁlISIS PRELIMINAR POR CADENA OPERATIVA DE LOS DESECHOS DE TALLA lítica del Complejo Ayacucho, Perú
}

Juan Yataco Capcha*

\section{Resumen}

El análisis del material lítico arqueológico del complejo Ayacucho de la cueva de Pikimachay correspondiente al Pleistoceno Final, ha permitido la identificación tipológica definida como desechos de talla, conformada i.e. núcleos desbastados, lascas primarias y secundarias y fragmentos de lascas obtenidas muy probable por percusión dura y/o blanda.

Este trabajo presenta los resultados de la aplicación de una ficha de registro métrico, efectuado bajo la metodología de "Cadena Operativa", con el objetivo de poder identificar medidas promedio y posibles orden de secuencia de talla lítica. Sin embargo, aún sabiendo que contamos con una evidencia mínima cualitativa de material lítico arqueológico procedente del Complejo Ayacucho, el resultado del análisis preliminar ha permitido al menos asociarlas, con tres momentos de talla lítica.

Palabras clave

Cadena Operativa, Pleistoceno final, Cueva de Pikimachay, Complejo Ayacucho.

\section{Abstract}

The analysis of archaeological lithic material from the Pikimachay cave at the Ayacucho Complex corresponding to the Final Pleistocene, have led to definite typological identifications such as core debris, first and secondary flakes, and fragments of slices which were most likely obtained through soft or hard hammer percussion.

This study shows the results of an analysis of metric measurements applied to the collection conducted under the methodology of "Chaine Opératoire" with the goal of identifying possible measures and the average order sequence for stone carving. However, knowing that we have minimal qualitative evidence on the archaeological lithic material from the Ayacucho Complex, the results of the preliminary analysis have demonstrated the association with three moments of stone carving.

\section{Keywords}

Chaine Operatoire, Pleistocene final, Pikimachay Cave, Ayacucho Complex.

\footnotetext{
* Licenciado en Arqueología de la Universidad Nacional Federico Villarreal y Curador Lítico del Museo de Arqueología y Antropología, Universidad Nacional Mayor de San Marcos.

Correo electrónico: capchajuan@gmail.com
} 


\section{INTRODUCCIÓN}

Se viene trabajando en el Museo de Arqueología y Antropología de la Universidad Nacional Mayor de San Marcos, la discriminación, conservación preventiva e inventario de los materiales líticos modificados de la colección del Proyecto Arqueológico Botánico Ayacucho, el cual fuera dirigido por el arqueólogo Richard MacNeish y su equipo de investigación (MacNeish 1969; 1979, MacNeish et al. 1970; 1980; 1981; 1983).

Durante los avances, hemos podido identificar y evaluar de manera preliminar los materiales en piedra modificados de la Cueva de Pikimachay, procedentes del estrato más temprano denominado como complejo Ayacucho, el cual esta conformado por las capas sucesivas "hl" y " $h$ ". A partir de la identificación de las piezas líticas y su asociación con el complejo Ayacucho, se han publicado algunos resultados preliminares (vide, León 2007: 218-225, Yataco 2008: 15-18, León et al. 2008: 34-37, León et al. 2009: 2-4).

Asimismo, habiendo realizado una revisión exhaustiva de las herramientas en piedra, hemos abordado el análisis de los desechos de talla lítica del complejo Ayacucho, aplicando la metodología de "cadena operativa". Esta categoría es entendida como el principio que engloba la adquisición, elaboración, uso y abandono de utillajes sobre distintas materias primas (Pelegrin 1995: 25, Inizan 1995: 14, Merino 1994: 435). Para ello, se ha elaborado las fichas de registro de material lítico en función de las cadenas operativas tal como lo propuesto por Pelegrin (Op. Cit.: 20-39). De este modo hemos podido identificar sensu lato tan solo "momentos" de los procesos técnicos de confección, las cuales han servido de guías, para organizarlas dentro de las actividades del proceso de talla y de este modo aproximarnos a entender la manufactura empleada sobre el material lítico arqueológico.

\section{Entorno Geográfico}

La cueva de Pikimachay, denominada también Cueva de La Pulga, se localiza según MacNeish a los 741'ㅇ' 'W y 133'30'S, en el departamento de Ayacucho, a unos $12 \mathrm{~km}$ al noroeste de la provincia de Huamanga y a una altura de 2,850 m.s.n.m. (MacNeish 1979: 5).

La topografía es accidentada, presentándose de forma llana a ondulada debido a los plegamientos y fallas geológicas de la zona y oscila de forma abrupta a escarpada hacia el lado este (INGEMMET 1995: 7).

Según la clasificación climática realizada por Koppen, el entorno geográfico de se enmarca con un clima templado moderado lluvioso, con temperaturas medias que oscilan entre $13^{\circ} \mathrm{C}$ a $15^{\circ} \mathrm{C}$ (cf. INGEMMET, 1995: 10; fig. 3a; 3b). La vegetación esta constituida por plantas que resisten las sequías e.g. cactáceas, tuna, huarango, quinuales e ichu. Por otro lado, con dirección suroeste a partir de la cueva y a unos $3 \mathrm{~km}$ se disectan los ríos Huarpa, Pongor y Cachi; siendo este último el principal colector de drenaje y afluente del río Mantaro (INGEMMET 1995: 8; 13).

\section{ANTECEDENTES}

La cueva de Pikimachay es uno de los yacimientos que más ha llamado la atención de los especialistas, debido a los hallazgos controversiales de presencia humana más temprana en el Perú.

En tres reportes se pueden encontrar los datos sobre los trabajos efectuados en este yacimiento (MacNeish, et al. 1980, 1981 y 1983); sin embargo, es sabido que estos reportes no se presentan bien fundamentados (Rick 1992: 16), asimismo es imposible tener en claro el comportamiento del material lítico arqueológico y su asociación con otras industrias líticas andinas (Dillehay 1985: 196). Por otro lado, muestras de carbono 14C tomadas de hueso también son puestas en tela de juicio debido a las imprecisio- 
nes del método de fechado y a sus asociaciones contextuales (e.g. Rick 1992: 13, Lynch 1990: 25; 1992: 255).

Finalmente, la mayor controversia radica en la presencia de supuestas piezas líticas modificadas en piedra, provenientes de los estratos inferiores de la cueva de Pikimachay, denominados como complejo "Pacaicasa" conformados por cuatro estratos definidos como " $i$, il, j y $k$ " (cf, MacNeish 1979: 21-29; MacNeish et al. 1983: 136-138) y el complejo "Ayacucho" conformado por los estratos " $h$ y $h 1$ " (cf, MacNeish 1979: 29-41). Sin embargo, reexaminando los materiales líticos podemos concluir que, el Complejo Pacaicasa carece de material arqueológico en piedra; empero, es en la fase Ayacucho donde se ha identificado un número representativo de material lítico antrópico.

\section{OBjetivos y MÉTOdo de LA INVESTIGACIÓN}

En primer lugar, habiendo identificado el material arqueológico lítico del complejo Ayacucho y sabiendo que carece de definiciones morfotecnológicas y menos aún, sin contar con el registro métrico respectivo hemos decidido abordar este problema.

Es necesario también aclarar que, la colección del proyecto Botánico Arqueológico Ayacucho no ha sido ajena a alteraciones en su estado de conservación. Sabiendo que por más de treinta años ha estado expuesta a diferentes agentes de deterioro en los viejos ambientes de la universidad San Marcos, hemos examinado las piezas líticas arqueológicas descartando y diferenciando las piezas con retoques moderno de las que presentan retoque arqueológico.

Finalmente, el registro descriptivo de los restos de talla arqueológica nos ha llevado a establecer medidas promedio para reproducir parte de la cadena operativa. Para lograr este objetivo, se ha elaborado las fichas de registro bajo la metodología francesa (Pelegrin 1995: 26-
32), las cuales toman en cuenta una serie de características métricas (e.g. porcentaje de córtex, ángulos de borde bifacial y de expulsión, etc.)

\section{Estrategia de la investigación}

La revisión general del material lítico ha sido el punto de inicio de esta investigación, seguido de la identificación preliminar de las piezas y descarte de los retoques no intencionales producidos casualmente. Sucesivamente, se procedió a elaborar el registro partiendo de los apuntes tomados. Se ha obtenido así, una primera división en dos grupos a las cuales hemos denominado: restos de talla y desechos núcleo. Una vez obtenido la separación de las piezas líticas por grupos, se continúo con el examen minucioso del material lítico con una lupa de 10X y $15 \mathrm{X}$ de aumento. Al abordar el estudio de las lascas, se pudo constatar que estas también presentaban ciertas características particulares, con la cual las hemos identificado e.g. fragmentos de Lascas Secundarias a Percusión Dura y Blanda y lascas completas (fragLSPD; fragLSPB). En el caso de los núcleos, también se ha visto permisible la elaboración de definiciones en dos series de tipos (e.g. núcleo desbastado y desecho de núcleo). A continuación, en función a la colección y bajo la metodología de "cadena operativa", se crearon las fichas para, núcleos y restos de talla. Estas fichas contemplan los rubros de mediciones, pesos, formas y tecnología.

El resultado de dicho análisis se ha vertido a una base de datos en el formato Access y/o Excel, para facilitar el manejo de la información. Con este grado de información, se procedió a la elaboración del presente informe.

\section{Ficha DE DOCUMENTACiÓN}

Las fichas de registro del material lítico aplicada sobre esta colección, presentan una serie de rubros, que sesu lato contiene lo siguiente: características métricas, tecnológicas, peso, for- 
mas y observaciones. En general, todas las fichas muestran los mismos parámetros para la obtención de la información básica.

En primer lugar, el encabezado, contiene lo datos del tipo de ficha y nombre de la colección. Seguido de un cuadro para la determinación del material lítico antrópico, luego medidas de longitud, peso, medidas angulares, descripciones de la superficie, etc. Una vez concluido el llenado de todos los recuadros que contiene la ficha, se realiza la determinación diagnostica de la pieza lítica (e.g. LSPD: lasca secundaria de percutor duro; LSPB: lasca secundaria de percutor blando; fragmento meso-distal de LSPD; desecho de núcleo, etc.)

Asimismo, esta colección presenta un numero secuencial a cada pieza lítica antrópica (e.g. L-0001, L-0002. L: Lítico; 0001: número de inventario de la pieza). Se ha empleado esta codificación para realizar la localización más sencilla de la pieza lítica en las cajas que conforman la colección del Proyecto Etnobotánico Ayacucho y obtener así la cuantificación real de la colección. También, se ha introducido los rubros de datos de análisis y materia prima, el cual comprende, la observación sensu strictu sobre el tipo de roca. Sin entrar en especificaciones precisas de la mineralogía.

\section{Estratigrafía del Complejo Ayacucho}

El denominado complejo Ayacucho, esta conformado por los capas " $h$ " y " $h 1$ ", es en estas capas en donde se han identificado artefactos líticos modificados por acción humana.

\section{Capa "h1"}

La capa " $h 1$ ", es la más profunda y se encuentra yaciendo por sobre el estrato " $i$ " del complejo Pacaicasa. Esta capa tiene un espesor que oscila entre 25 y $50 \mathrm{~cm}$. Asimismo, se han registrado seis áreas de actividad, conteniendo evidencias de talla lítica en supuesta asociación con huesos de animales del pleistoceno (MacNeish 1979: 29-33).

\section{Capa " $h$ "}

Con un espesor de $30 \mathrm{~cm}$ y superpuesta al estrato " $h 1$ ", en esta capa se han registrado siete áreas de actividad y la presencia de dos fogones que no cuenta con descripciones detalladas. También se señala la presencia de restos óseos de animales pleistocenicos al parecer según MacNeish con claras evidencias que muestran su procesamiento por medio de los artefactos en piedra (MacNeish 1979: 33-40).

\section{El material lítico del Complejo Ayacucho}

\subsection{Los Núcleos del Complejo Ayacucho}

Los núcleos son las piezas líticas con alto potencial de información durante el análisis tecnológico. Nos brinda información sobre la puesta en forma practicada, la materia prima seleccionada, la organización de la superficie del desbastado y hasta circunstancias de abandono (Pelegrin 1995: 109).

Dos piezas han sido reconocidas, una de ellas ha sido determinada como núcleo desbastado (Fig. H) procedente de la capa "hl", seguida de un desecho de núcleo (Fig. Q) localizada en la capa " $h$ " del complejo Ayacucho.

\section{Análisis del núcleo de la capa "h1"}

Determinado como un núcleo desbastado (Fig. $\mathrm{H})$, se trata de una pieza sobre cuarzo con una pequeña inclusión de tufo volcánico, pátina opaca y color amarillo ocre. Es de forma ortogonal con presencia de 50\% de córtex o más en dos de sus facetas. Tiene como número de catálogo Ac100 210-1-1nn. SS53, proviene de la cuadricula S21E4, zona h1 (MacNeish et al., 1980: 193). Sobre sus facetas se observan negativos profundos no organizados con dirección centrípeta, los cuales han sido realizados posiblemente por percusión con percutor duro, sobre un plano de percusión cortical (Esquema 1, 1A). Sus longitud ancho y espesor es de 43×37×26 mm 

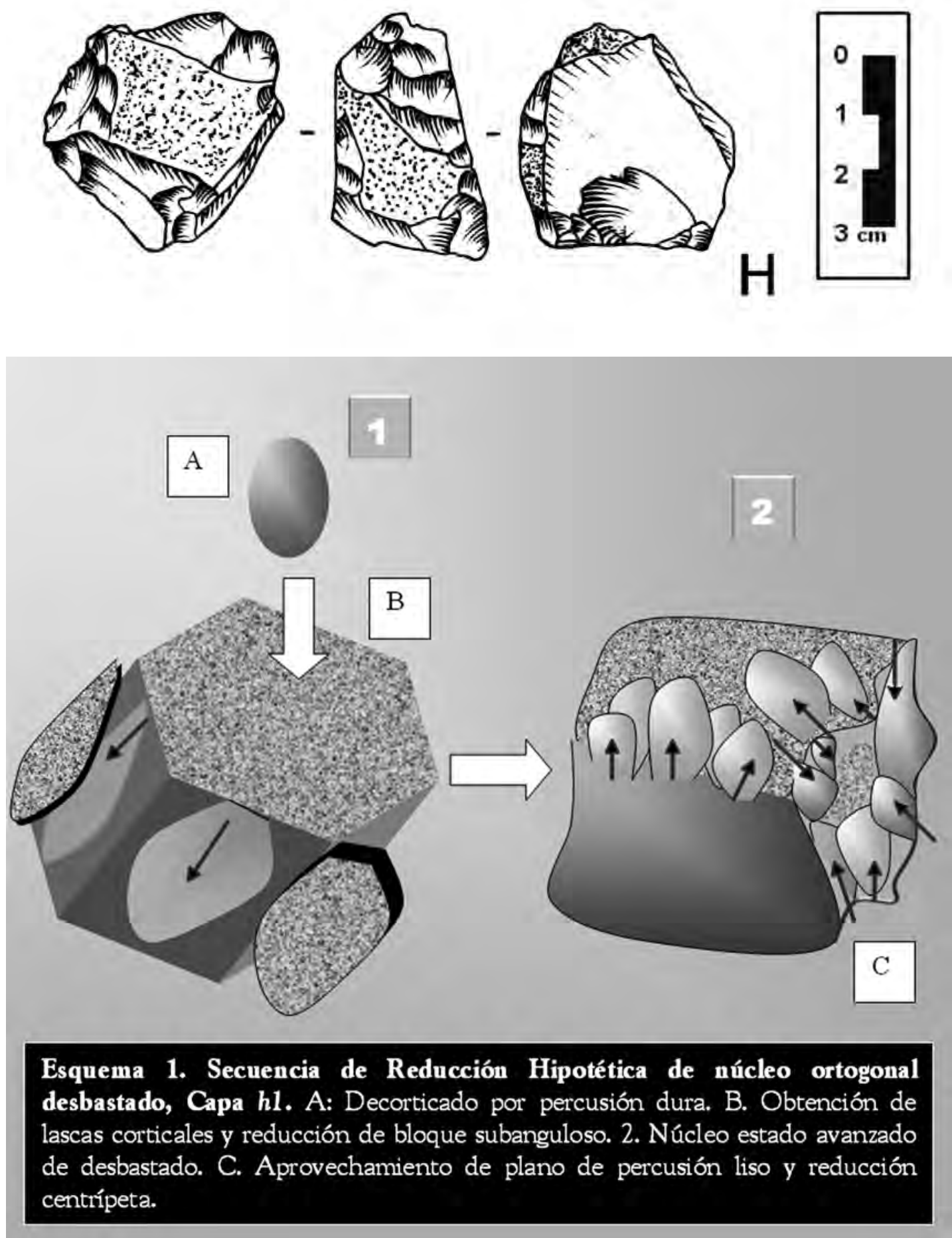
y peso de 54.69 gr. El ángulo de plataforma de expulsión se ha determinado en un ángulo de 90 .

La presencia de córtex oscila entre un 50\% y $80 \%$ y sus medidas para dos de sus facetas del núcleo desbastado de la capa $h 1$, hacen pesar en la selección de un blank de forma angulosa ortogonal (pequeño bloque subanguloso) con un plano de percusión lisa, y muy posible para la obtención de lascas corticales (Esquema 1, 2B). Como se ha dicho anteriormente, se observa un plano de percusión liso cuyos ángulo de plataforma miden $90^{\circ}$ y la disposición de los negativos originados por percusión dura se dispone de forma centrípeta en el núcleo desbastado de la capa hl (Esquema 1, 2C).

\section{Análisis del núcleo de la capa " $h$ "}

Se trata, de un fragmento de núcleo desbastado sobre una roca de cuarzo lechoso, pátina opaca y de color externo amarillo ocre (Fig. Q). Con rotulo Ac100 281-III-nn1. SS21, proviene de la zona h, cuadricula S25-26E7 (cf. MacNeish et al. 1980: 196). Presenta una forma rectangular irregular, debido a los desprendimientos generados por impactos de probable percutor duro en toda su superficie. La selección del blank hace pensar en un bloque también subanguloso (Esquema 2, B). La organización de sus negativos de disposición centrípeta y longitudinal en sus facetas y un plano de percusión lisa a partir de una lasca reflejada (vide, Figura Q, dibujo extremo derecho) puede permitirnos asociarlo con un trozo de lasca o desecho de bifaz relativamente grande y burdo que esta siendo nuevamente reutilizada. Podría tratarse en este caso de un desecho procedente de faces de reducción media que ha sido abandonado por presentar una diaclasa que se dispone de forma lateral en la pieza (Cf. Fig Q, dibujo central), lo cual ha ocasionado que la extracción de lascas no sea del todo exitosa.

Se observa al menos dos planos de percusión lisos con plataformas de expulsión que oscilan entre 80 y 90 (Esquema 2, C), también obtenido por plausible percusión dura (Esquema 2, A). Su longitud es de $51 \mathrm{~mm}$, ancho de 38 mm y espesor de 26. El peso es de 54.03 gr.
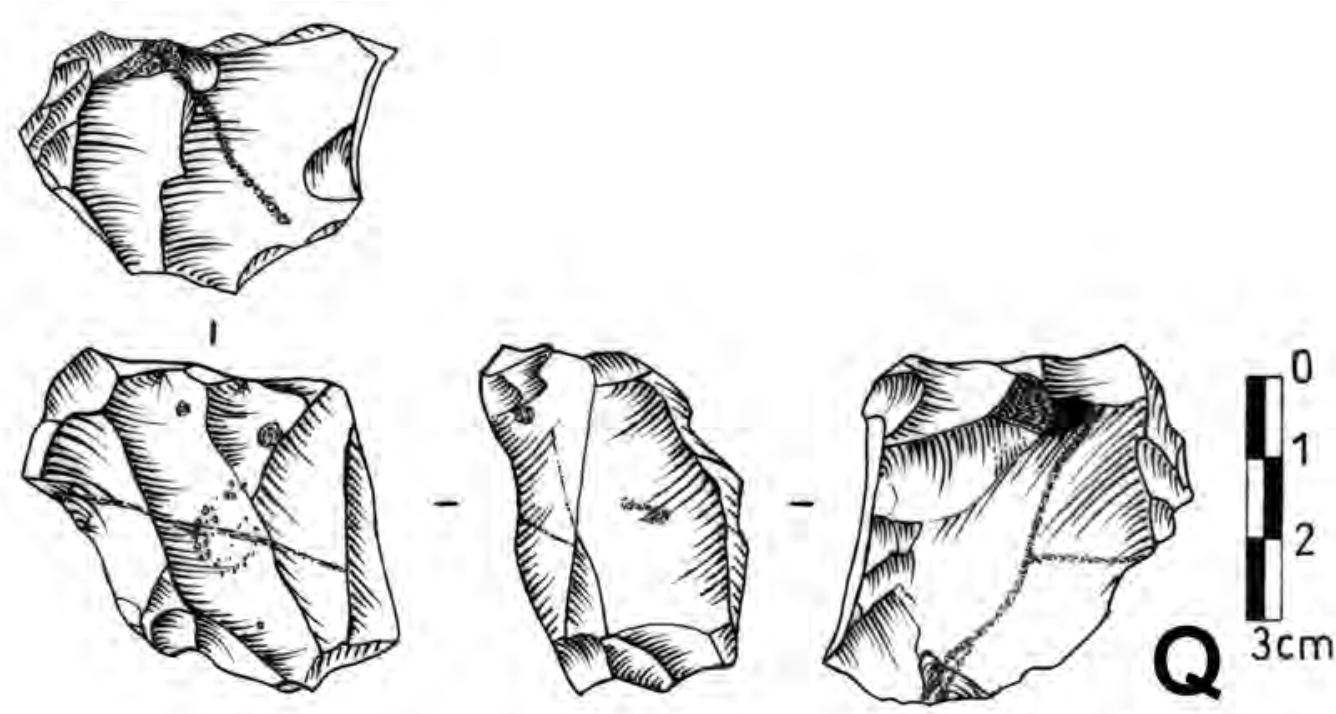

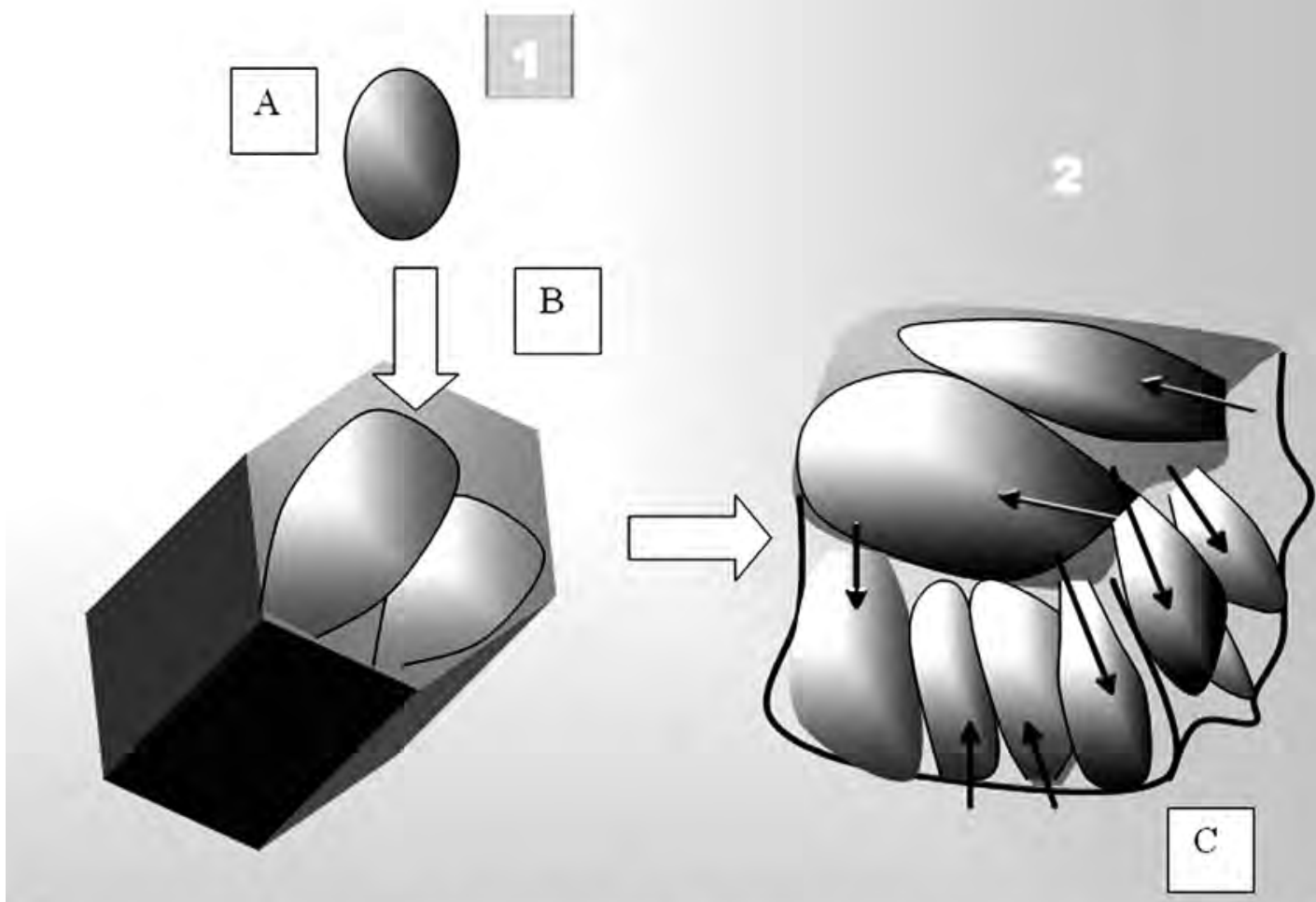

Esquema 2. Secuencia de Reducción Hipotética de núcleo desbastado, con plano de percusión liso, Capa $\boldsymbol{h}$. A: Reducción por percusión dura. B. reducción de núcleo ortogonal. 2. Núcleo sin presencia de córtex. C. Aprovechamiento de plano de percusión liso y negativos paralelos de posibles laminillas.

\subsection{Los restos de talla del Complejo} Ayacucho

Los desechos de talla son los materiales arqueológicos más importantes desde el punto de vista cualitativo, para ser analizadas bajo el principio de las cadenas operativas. Asimismo, estos han sido clasificados de a cuerdo a la técnica empleada. En este grupo se han incluido todos los productos resultantes de la elaboración de los artefactos líticos, i.e. lascas y formas derivadas y desechos de talla propiamente.

Diez piezas conforman el material documentado; cuatro desechos provienen de la zona
" $h 1$ " y seis proceden de la capa " $h$ ", ambas capas conforman el complejo Ayacucho. Para abordar este análisis, nos parece poco significativo el segregar estas capas, sin embargo, por rigurosidad científica vamos a analizar estas piezas distinguiéndolas por su procedencia estratigráfica.

\section{A. Análisis de los desechos de talla de la capa "h1"}

En primer lugar, los desechos de la capa más profunda " $h 1$ ", está conformado por la existencia de un fragmento longitudinal de lasca del tipo siret, obtenida por probable percusión dura 
y posiblemente modificada (Fig. J). Sin embargo, debido a lo agudo del borde modificado y de su posible fractura antropogénica y al estado de conservación de la piezas, hemos preferido catalogarla entre los restos de talla. Se encuentra modificada sobre un tufo volcánico de buena fractura, pátina opaca y color granate. Tiene como número de catálogo Ac100 231-7-d3// SS10, procede de la cuadricula S18E5-5 ha sido asociada al complejo Ayacucho (MacNeish et al. 1980: 190). Su longitud es de $37 \mathrm{~mm}$, ancho $17 \mathrm{~mm}$ y espesor de $10 \mathrm{~mm}$ y su peso de 6.63 gr. Su silueta es rectangular y vistas de lateral y perfil se dispone de forma trapezoidal. No presenta talón y su bulbo es difuso. No hay presencia de córtex y sobre su cara ventral se observa la presencia de un negativo lateral la cual fue realizada posterior a su extracción, lo cual indica su estado avanzado de extracción durante la talla (vide, esquema 3, A). Asimismo, en la cara dorsal, se observan al menos tres negativos que se disponen de forma vertical lo cual apoyaría la idea anteriormente mencionada (vide, esquema 3, B). La tecnología empleada en su obtención ha sido la percusión dura, en el esquema 3, letra C, podemos apreciar su terminación distal en $45^{\circ}$ relativamente gruesa y de buen filo puede indicarnos que su extracción fue relativamente exitosa. La fractura longitudinal ha sido al parecer por causas tecnológicas debido al exceso de golpe al momento de la reducción.

Seguidamente, sobre un silex achocolatado tenemos la presencia de un fragmento mesial de lasca secundaria a percusión dura (FragMesialLSPD). Ha sido identificado con el número de catálogo Ac100 152-?-Dd. SS53 y de la cuadricula S21E6 (cf. MacNeish et al. 1980: 193). Sobre una piedra de color marrón claro y pátina brillosa. Tiene esta pieza una longitud de $37 \mathrm{~mm}$, ancho de $30 \mathrm{~mm}$ y espesor de 16 $\mathrm{mm}$. Sus formas trapezoidales vistas de silueta y sección además de su forma rectangular vista de perfil, solo hace pensar en un fragmento de lasca burda, gruesa y no exitosa. Su peso de 16.78 gr. también puede confirmar lo dicho. Sobre su cara ventral y hacia el extremo distal se observan cuatro negativos, los cuales han modificado la fractura muy posible tecnológica (Fig. K). Hacia su extremo proximal, se observa una clara fractura transversal de origen tecnológico en terminación en lengüeta, lo cual muestra de manera plausible su fractura por percusión dura.

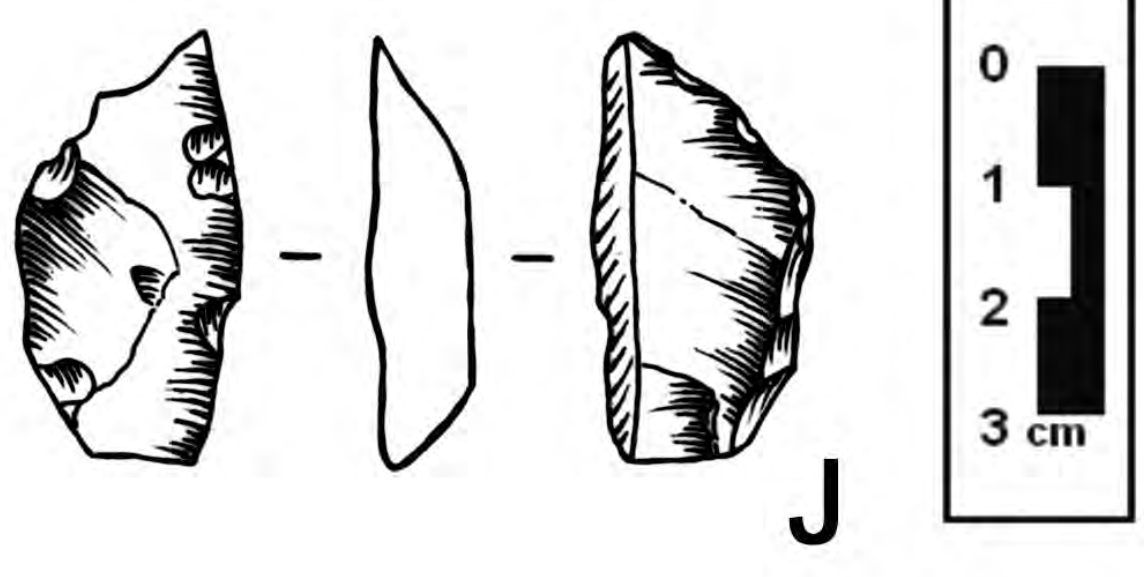




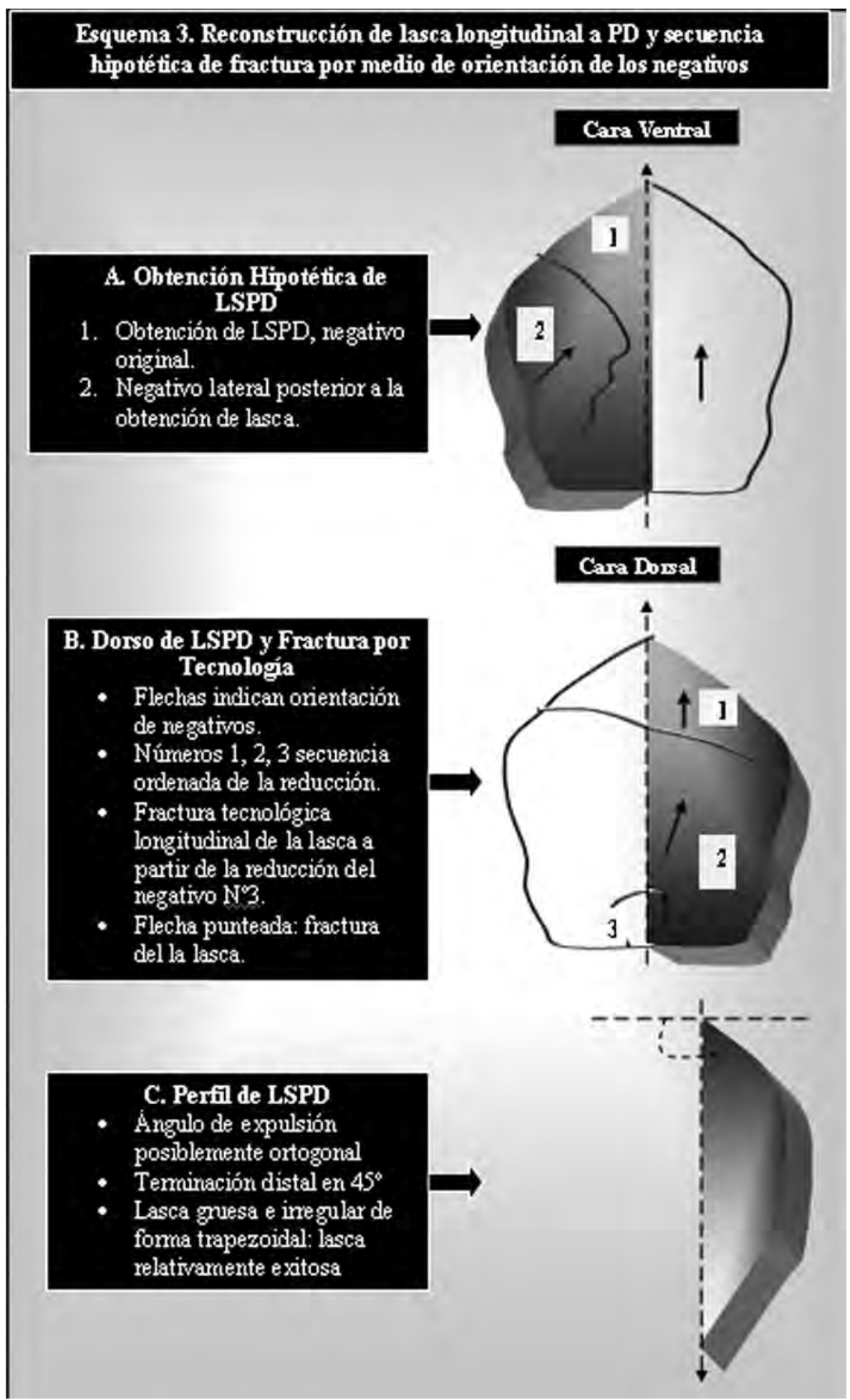


Asimismo, tres negativos dos longitudinal y uno lateral pronunciados concuerdan con la típica reducción por percutor duro, muy posible en etapas iniciales de talla.

Otros dos fragmentos no determinados, uno de ellos modificada sobre un tufo volcánico de forma tetraédrica, reducida a percusión dura y un segundo que presenta evidencias de haber sido expuesta al fuego, han sido halladas.

El desecho tetraédrico, tiene número de catálogo Ac100 161-VII y fue hallada en la cuadricula S21-22E5 (cf. MacNeish et al. 1980: 199, fig. 5-6; MacNeish 1979: 45, fig.23, no9). Ha sido publicado como una pieza con golpe de buril. Sin embargo, nosotros pensamos que más bien se trata de un desecho con evidencias de algunos golpes a percusión dura en su super- ficie. Tiene una longitud de $70 \mathrm{~mm}$ y con un ancho y espesor de $22 \mathrm{~mm}$. Su peso es de 37.10 gr presenta entre 5 a 6 negativos distribuidos en sus cuatro facetas, sin mostrar mayores datos. La tecnología empleada es el de la percusión con percutor duro.

La ultima pieza hallada de esta capa proviene de la cuadricula S18E5-5, presenta el rotulo Ac100 231-VII-ee. SS10 (cf. MacNeish et al. 1980: 190). Como se ha mencionado presenta evidencias de haber estado expuesta al fuego. Se trata de un tufo volcánico sedimentario que oscila de un color plomo a crema. Su longitud es de $36 \mathrm{~mm}$, ancho de $22 \mathrm{~mm}$ y espesor de $15 \mathrm{~mm}$. Su peso es de $7.81 \mathrm{gr}$. En una de sus facetas presenta córtex lo cual pone evidencia que su elección ha sido un blank anguloso. Su silueta es de
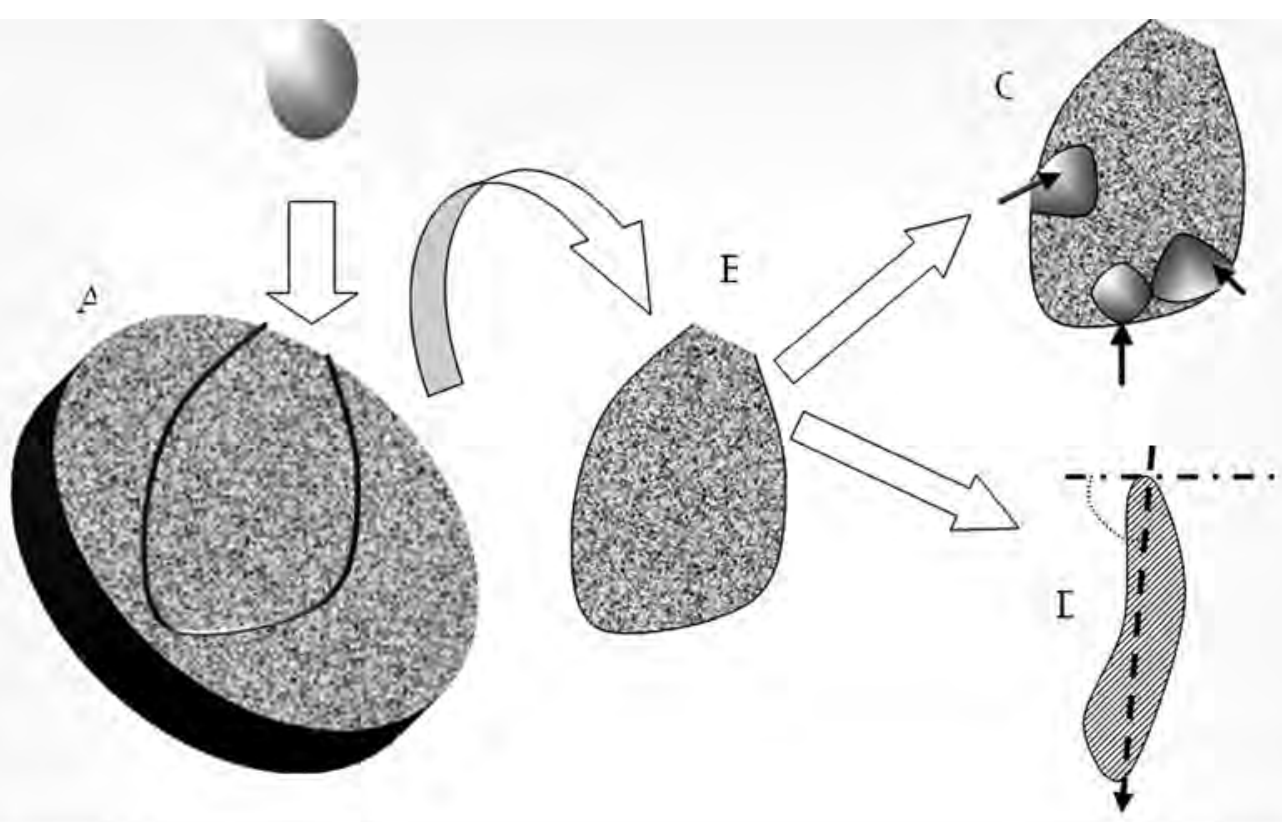

\section{Esquema 4. Secuencia de reducción y modificación de una lasca secundaria} de guija, estadio primario de decorticado.

A. Reducción por percusión dura sobre guija (rodado).

B. Obtención de lasca primaria

C. Reducción por percusión dura de lasca primaria

D. Vista de perfil de lasca con promedio de $80^{\circ}$ expulsión. 
forma rectangular, triangular irregular vista de perfil y de sección paralelepípedo.

\section{B. Análisis de los desechos de talla de la capa " $h$ ".}

Seguidamente en la capa " $h$ " superpuesta por sobre la capa " $h l$ " se han identificado dos lascas secundarias completas a percusión dura (LSPD), ambas piezas modificadas sobre tufo volcánico sedimentario, son de muy buena silicificación y fractura. sobre la cara dorsal tres negativos centrípetos y dos nervaduras ligeramente pronunciadas (Esquema 4, C).

La siguiente LSPD (Fig. M), procedente de la cuadricula S19E4 y con rotulo Ac100 165. VIII-H, ha sido mejor definida. Esta presenta una coloración que oscila entre beige a negro y de pátina opaca. Su longitud es de $42 \mathrm{~mm}$, ancho de $51 \mathrm{~mm}$, espesor de $9 \mathrm{~mm}$ y peso de 15.08 gr. El punto de impacto es claro y su ángulo de expulsión es de 75으, siendo típica de reducción

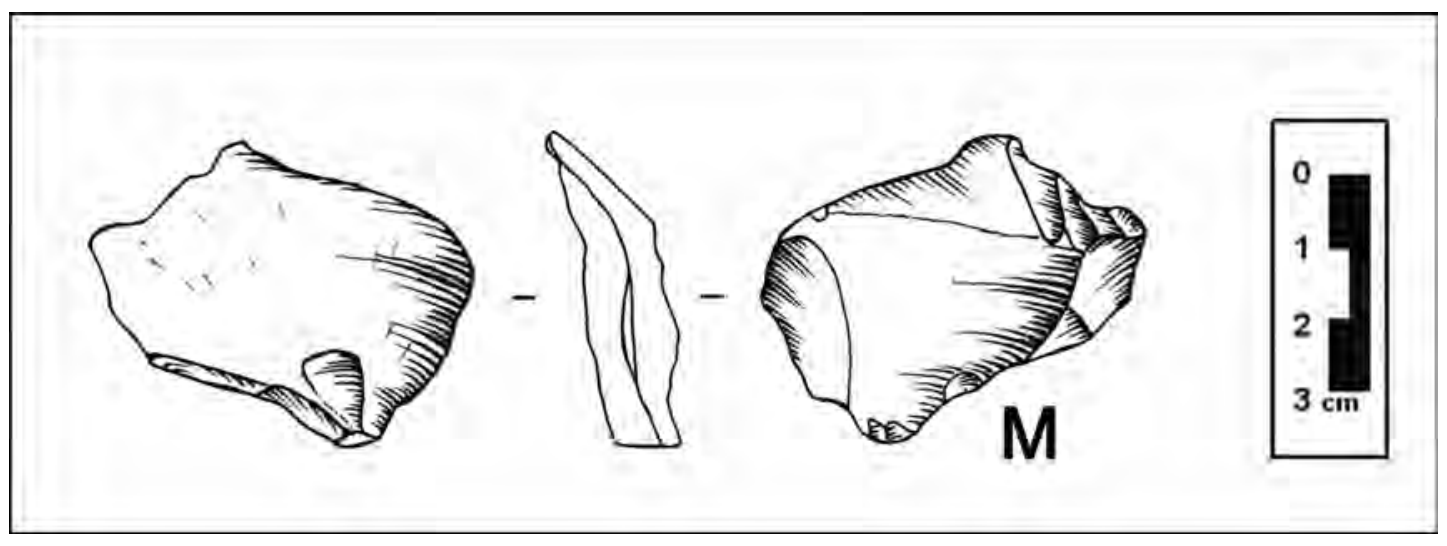

Una de las lascas obtenidas por percusión dura de la cuadricula S23E7 (Esquema 4, A), presenta el número de catálogo Ac100 153-2-e4. SS56 (cf. MacNeish et al. 1980: 198). Presenta una coloración granate y pátina brillosa debido a la presencia de córtex en un 90\% (Esquema 4, B). Por la naturaleza del córtex sabemos que se ha hecho la selección de un blank de guija (Esquema 4, A), con un ángulo de expulsión aproximado de $80^{\circ}$ (Esquema 4, D). Su longitud es de $71 \mathrm{~mm}$, ancho de $54 \mathrm{~mm}$ y espesor de 16 $\mathrm{mm}$. Su peso a comparación de otras lascas ha sido de 69.22 gr., lo cual devela su estadio primario de decorticado. Su silueta, perfil y sección de forma rectangular no hace más que confirmar la obtención burda típica de talla inicial. El punto de impacto y el talón no son muy claros debido al estado de intemperismo. Presenta bifacial, su terminación distal termina en un ángulo que oscila entre 45 a 50․ Su silueta rectangular y perfil y sección cóncavo convexo confirman su procedencia bifacial. El tipo de talón es liso y ligeramente fracturado debido al golpe de impacto, además es de forma rectangular irregular, el bulbo es expandido sobre la ventral. En la dorsal se observan un negativo central longitudinal y siete negativos laterales por percusión dura. Finalmente, esta lasca presenta una falla por tecnología, siendo ligeramente reflejada.

Por otro lado, otros dos fragmentos han sido determinados como fragmentos meso proximales de lasca a percusión dura (FragMesoProxLSPD), han sido halladas con los números de catálogos Ac100 224-1. SS10 de la cuadricula S22E3 y Ac100 226-4dd. SS10, cuadricula S22E9 (cf. MacNeish et al. 1980: 190). Sus diferencias sig- 
nificativas han sido la variación de sus colores y el tipo de materia prima. Pues la primera se trata de un tufo volcánico de color granate y la segunda de un silex azulado. Ambos presentan pátina opaca, sus longitudes tienen una media de $29 \mathrm{~mm}$, ancho de $38 \mathrm{~mm}$ y espesor también con una media de $12.5 \mathrm{~mm}$. Sus pesos han tenido una media de $12.43 \mathrm{gr}$. Su silueta, perfil y sección oscilan entre las formas trapezoidal, triangular y rectangular. Sus bulbos son difusos y puntos de impacto claros. El ángulo de expulsión de estas lascas tiene una media que oscila entre $70^{\circ}$ a 75‥ El tipo de talón sobre la pieza de tufo volcánico es cortical y de forma triangular, midiendo el talón $29 \mathrm{~mm}$ de longitud y $12 \mathrm{~mm}$ de ancho. La presencia de córtex ha develado nuevamente la predilección de una guija para la reducción bifacial, asimismo esto hace juzgar que estamos frente a una lasca del estadio de puesta en forma, sobre su dorsal cuatro negativos centrípetos y tres nervaduras pronunciadas hacen pensar en la reducción de piezas bifaciales.

Caso contrario es en el talón de lasca tallada sobre silex azulado. Pues el talón ha sido preferentemente liso de forma biconvexa y con medidas de $13 \mathrm{~mm}$ por $5 \mathrm{~mm}$, como también sobre su cara dorsal solo se cuenta con la presencia de un negativo central.

En ambas piezas la fractura transversal ha sido determinada en terminación en lengüeta, debido a causa tecnológica muy probable ocasionada por percusión dura.

También se ha documentado, un fragmento meso proximal de lasca a percusión blanda (FragMesoProxLSPB) y un fragmento meso distal de lasca primaria a percusión dura (FragMesoDistalLPPD).

La materia prima del primer resto de talla, ha sido determinada como un cuarzo lechoso, de color blanco y pátina opaca. Con número de catálogo Ac100 257-III-1-2. SS10 procedente de la cuadricula S19E7 (cf. MacNeish et al. 1980: 190); tiene una longitud de $14 \mathrm{~mm}$, ancho y espesor de $3 \mathrm{~mm}$ su peso de 0.75 gr y ángulo de expulsión de 55ํㅡ típica en lasca de reducción bifacial. Su silueta y perfil de forma rectangular, sección triangular, talón liso de forma triangular, que mide $3 \mathrm{~mm}$ de largo por $2 \mathrm{~mm}$ de ancho hacen asociarla con la percusión blanda. Se trata de una lasca de avanzado estadio de talla (posible regularización) debido a no contar con córtex y a lo delgado de su espesor y bulbo difuso. Además, a esto se suma los datos de la cara dorsal de este resto de talla, el cual se define por la presencia de dos negativos longitudinales y una nervadura central, lo cual permite asociarla con un blank preparado para la extracción de micro laminillas. Finalmente la fractura transversal de este desecho se ha debido a fallas tecnológicas cuya terminación se presenta en lengüeta.

Sobre el fragmento meso distal de lasca primaria, con rotulo Ac100 221-?-d. SS6, de la cuadricula S18E4, zona h, se encuentra sobre un basalto de buena fractura, su longitud, ancho y espesor ha sido de $42 \times 48 \times 20 \mathrm{~mm}$ y un peso de 32.59 gr. Sobre la cara dorsal la presencia de córtex ha hecho relacionarla con la selección de un blank anguloso. Su silueta y perfil rectangular, asimismo como su sección trapezoidal como por sus medidas y peso denotan que se trata de una lasca de primera generación.

\section{CONCLUSIÓN Y DISCUSIÓN}

Si bien, no contamos con una cantidad suficiente de débitage para poder establecer estadios de talla y en eso somos completamente concientes, los hallazgos preliminares de estos materiales arqueológicos en piedra, del complejo Ayacucho y su estudio por cadenas operativas, nos ha permitido por el momento asociarlas sensu lato, con tres momentos de talla.

El análisis de dos núcleos y restos de talla, han develado la preferencia y el conocimiento del tallador prehistórico en seleccionar materia prima de buena silicificación. En primer lugar, la predilección se ha efectuado sobre cuarzo, silex, 


\section{Esquema 5. Momentos de talla detectados del Complejo Ayacucho.}

A. Decorticado: Elección de blanks ortogonal y guija. Obtención de lascas corticales burdas, con ángulos $80^{\circ}$ a $90^{\circ}$. B. Puesta en forma: Núcleo y/o útil desbastado, se obtienen LSPD sin presencia de córtex, talón liso y cortical, ángulo de expulsión oscila $70^{\circ}-75^{\circ}$. C. Posible Regularización?: lasca de regularización y corrección de irregularidades.

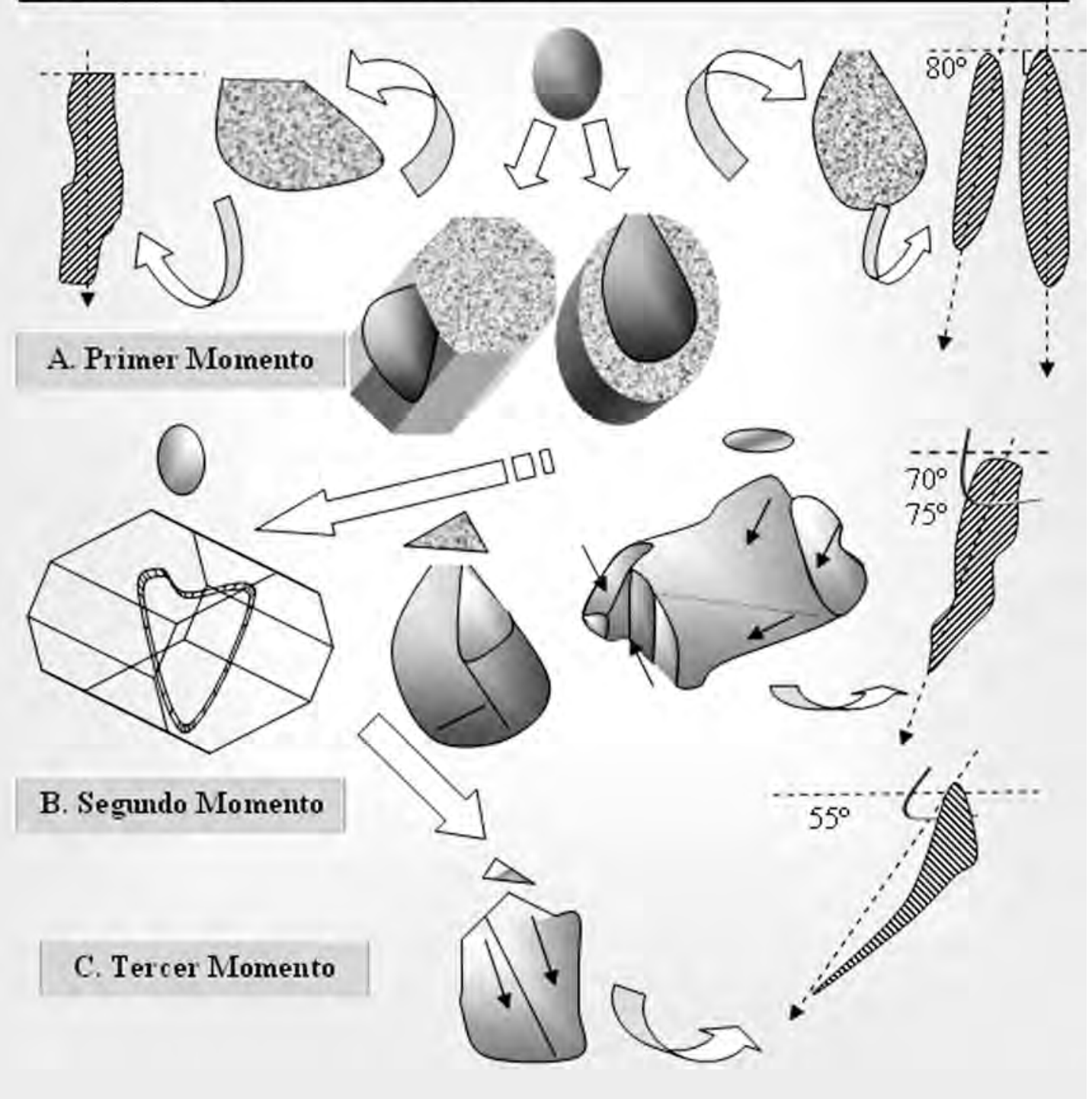

arenisca cuarzosa, basalto y tufo volcánico. No llama la atención que la presencia de cuarzo, tufo volcánico sedimentario y fenocristales sean de origen local, pues estas están presentes en las formaciones Ayacucho (Nm-Ay1) y Huarí (NpHu) (INGEMMET, 1995: 37-41; 50-54).

En primer lugar, (Esquema 5, A) según las evidencias expuestas nos permite argüir que la 
selección de gravas y pequeños bloques de formas subangulas a subredondeadas (guijas) ha sido las predilectas para el decorticado por probable percusión dura. En este caso, un núcleo con presencia de córtex y con ángulo de plataforma lisa de expulsión que mide $90^{\circ}$, puede haberse elegido y aprovechado para extracción de lascas para su posterior uso. Otra evidencia es la selección de una blank con forma redondeada para la obtención de lascas corticales, en este caso se ha tenido una lasca primaria de percusión dura con un peso promedio de 69.22 gr, con ángulos de expulsión que oscilan entre $80^{\circ}$ a $90^{\circ}$ y nos permite por el momento, asociarlos a este primer momento de talla.

El segundo momento de talla (Esquema 5, B), ha sido definido por la presencia de desecho de núcleo que evoca a un trozo burdo de lasca, de reducción bifacial y muy posible re-usada, luego abandonada por la presencia de una diaclasa lateral que ha echado a perder la reducción. Asimismo, lascas completas, con un peso que oscila entre 15.08 gr y 16.78 gr y con un promedio de 12.83 gr con ausencia de córtex en sus caras dorsales permiten juzgarlas con eventos de talla media. Estas han sido obtenidas por percusión dura con ángulos de expulsión que oscilan entre $75^{\circ}$ a $80^{\circ}$. Otro indicador de este momento de talla es que una lasca conserva al menos un talón cortical de forma triangular que mide $29 \mathrm{~mm}$ de longitud por $12 \mathrm{~mm}$ de ancho. Mientras que el segundo posee un talón liso de forma biconvexa de $13 \mathrm{~mm}$ de longitud y $5 \mathrm{~mm}$ de ancho, lo cual permite asociarlas muy posible con tecnología percutor duro. Además, sus formas de preferencia triangular irregular y longitud que oscila entre $42 \mathrm{~mm}$ a $37 \mathrm{~mm}$ con un promedio de $38.6 \mathrm{~mm}$, ancho que varía de 51 $\mathrm{mm}$ a $17 \mathrm{~mm}$ con un promedio de $32.6 \mathrm{~mm}$ y espesor que fluctúa entre $9 \mathrm{~mm}$ y $16 \mathrm{~mm}$ con un promedio de 11.6, evidencian muy posible que son desechos obtenidos de la puesta en forma de talla bifacial.
Finalmente, un posible tercer momento detectado (Esquema 5, C), que puede ser denominado, como de "regularización", podría ser juzgado por la presencia de un fragmento meso proximal de lasca obtenida a percusión blanda. Su largo de $14 \mathrm{~mm}$, y sobre todo su ancho y espesor de $3 \mathrm{~mm}$ la delata como una lasca completamente delgada, lo cual creemos es posible asociarla con percusión blanda. También las medidas de $2 \mathrm{~mm}$ por $3 \mathrm{~mm}$ en su talón liso, dos negativos longitudinales y ángulo de expulsión de 55을 hacen asociarla con la fase avanzada de talla.

Por lo tanto, concluimos que los desechos de talla analizados ya sean por sus formas, tipo de materia prima, orden de negativos, peso, ángulos, etc; pueden estar indicando actividades relacionadas con la reducción de núcleos y útiles de talla bifacial.

\section{REFERENCIAS}

Dillehay, T. D.

1985 A Regional Perspective of Preceramic Times in the Central Andes. Reviews in Anthropology 12(3): 193-205

Inizan, Marie-Louise, Michèle Reduron-Ballinger, Hélène Roche y Jacques Tixier

1995 Technologie de la Pierre Taillée. Tome 4. CREP. France

INSTITUTO GEOLÓGICO MINERO Y METALÚRGICO (INGEMMET)

1995 Geología del Cuadrángulo de Ayacucho. Hojas: 27-ñ. En: Boletín №61. Serie A: Carta Geológica Nacional

León, Elmo

2007 Orígenes Humanos en los Andes del Perú. Universidad San Martín de Porres. Escuela profesional de Turismo y Hoteleria.

León, Elmo y Juan Yataco

2008 New Analysis of Lithic Artifacts from the Ayacucho Complex, Perú. En: Current Research in The Pleistocene. Volume 25. 
2009 Nuevo Análisis de los artefactos líticos del Complejo Ayacucho, Perú. En: Boletín del Museo de Arqueología y Antropología, UNMSM. Año 11, Enero-Marzo 2009.

Lynch, Thomas

1990 Glacial-Age Man in South America? A Critical Review. En: American Antiquity, 55(1), 1990, pp.13-36.

1992 La Realidad del Concepto Paleoindio en contra de las propuestas de MacNeish, Bryan y Gruhn. En: Revista de Arqueología Americana, №5, pp. 249261. Instituto Panamericano de Geografía e Historia. Mexico. D. F.

MacNeish, Richard

1969 First annual Report of the Ayacucho Archaeological-Botanical Project. Robert S. Peabody Foundation for Archaeology. Phillips Academy Andover, Massachusetts. Published by the foundation, $\mathrm{n}^{\mathrm{O}} 1$. Andover.

1979 The Early Man Remains from Pikimachay cave, Ayacucho Basin, Highland Perú. En: Pre-Llano Cultures of the Americas Paradoxes and Possibilities. Robert L. Humprey y Dennis Stanford, editors; pp. 1-47. Anthropological Society of Washington.

MacNeish, Richard, Antoinette Nekel-Terner \& Angel García

1970 Second Annual Report of the Ayacucho Archaeological-Botanical Project. Robert S. Peabody Foundation for Archaeology. Phillips Academy. Published by the foundation, n. -2 . Andover.

MacNeish, Richard, Robert Vierra, Antoinette Nekel-Terner \& Carl J. Phagan

1980 Prehistory of the Ayacucho Basin, Perú. Volume III. Nonceramic Artifacts. Robert S. Peabody Foundation for Archaeology. The University of Michigan Press. Ann Arbor.
MacNeish, Richard, Angel Garcia, Luis Lumbreras, Robert Vierra \& Antoinette Nekel-Terner

1981 Prehistory of the Ayacucho Basin, Perú. Volume II. Excavations and Chronology. Robert S. Peabody Foundation for Archaeology. The University of Michigan Press. Ann Arbor.

MacNeish, Richard, Robert Vierra, Antoinette Nekel-Terner, Rochelle Lurie \& Angel Garcia

1983 Prehistory of the Ayacucho Basin, Perú. Volume IV. The Preceramic Way of Life. Robert S. Peabody Foundation for Archaeology. The University of Michigan Press. Ann Arbor.

Merino, José Ma

1994 Tipología Lítica. Munibe (Antropología-Arkeoplogia). Suplemento n⒐ Sociedad de Ciencias Aranzadi.

Pelegrin, Jacques

1995 Technologie Lithique: Le Châtelperronien de Roc de Combe (Lot) et de la Côte (Dordogne). Cahiers du Quaternaire 20. CNRS Editions. Paris.

Rick, John

1992 The character and context of highland preceramic society. En: Peruvian Prehistory. An overview of Pre-Inca and Inca society. Cambridge University.

Yataco, Juan

2008 La cueva de Pikimachay y los posibles restos más antiguos del Perú: avance preliminar a partir de la discriminación lítica. En: Boletín del Museo de Arqueología y Antropología, UNMSM. Año 10, abril-junio 2008. 